\title{
A one-pot procedure for the synthesis of novel pyran-fused dicyanoanilines via a triethylamine/aqueous mediated pseudo four-component reaction
}

\author{
M. Saeed Abaee,* Soodabeh Forghani, Mohammad M. Mojtahedi, \\ and Atefeh Hadizadeh \\ Department of Organic Chemistry and Natural Products, Chemistry and Chemical \\ Engineering Research Center of Iran, Pajouhesh Blvd, 17th Km Tehran-Karaj Highway, \\ P.O.Box 14335-186, Tehran, Iran \\ E-mail:abaee@ccerci.ac.ir
}

This article is dedicated to Professor M. R. Saidi in honour of his 72 nd birthday

DOI: https://doi.org/10.24820/ark.5550190.p009.667

\begin{abstract}
A facile pseudo four-component process was developed for the synthesis of dicyanoaniline derivatives fused to pyran ring. Reactions took place in the presence of triethylamine via a one-pot combination of pyran-4-one 1 with various aldehydes and two equivalents of malononitrile in water. Thus, novel products 4 were obtained in high yields after $5-7 \mathrm{~h}$ mixing at $40{ }^{\circ} \mathrm{C}$. After completion of the reactions, products solidified in the mixture spontaneously. This allowed an easy separation of the products and avoided costly chromatographic purifications.
\end{abstract}

Keywords: Dicyanoanilines, isochroman, pyran-4-one, aqueous conditions, one-pot reaction

\section{Introduction}

In a multicomponent reaction (MCR), a minimum of three reactants combine in a one-pot process and directly lead to various libraries of products and target molecules. ${ }^{1-3}$ This feature has turned the multicomponent reactions into a one of the most useful strategies to design modern synthetic plans ${ }^{4-5}$ and sustainable chemical procedures. ${ }^{6-7}$ Dicyanoanilines demonstrate high quantum yield optical activities ${ }^{8-9}$ due to having acceptor-donor-acceptor (A-D-A) sequence of functionalities. Search in the literature shows that the existing methods for the preparation of these structures mainly go through MCR routes, ${ }^{10-11}$ because 
the MCR-based methods are more efficient than their equivalent stepwise processes. ${ }^{12-14}$ In addition, the fluorescence features of dicyanoanilines make them attractive and as a result continuous efforts are devoted by synthetic chemists in recent years to prepare diverse groups of dicyanoanilines ${ }^{15-20}$ and more studies are underway.

Pyran-4-one 1 is an important six-membered heterocyclic compound which is used in the synthesis of various heterocycles containing the pyran unit, ${ }^{21}$ possessing diverse biological features, ${ }^{22}$ and being part of the structure of several natural products. ${ }^{23}$ Therefore, there is always demands for developing new procedures to prepare various structures containing the pyran unit as the key fragment. We are interested in the chemistry of pyran-4-one. In this context, we have studied the synthetic applications of $\mathbf{1}$ in several investigations. ${ }^{24-28}$ In continuation, now we present a convenient and practical procedure by which ketone $\mathbf{1}$ undergoes a one-pot combination with aldehydes $\mathbf{2}$ and malononitrile $\mathbf{3}$. It should be noted that malononitrile is a very convenient reagent for many MCRs due to having a reactive methylene and two cyano groups to build several carbon-carbon and carbon-heteroatom bonds simoltaneously. ${ }^{29}$ Thus, a pseudo four-component process takes place and a new series of dicyanoanilines 4 is obtained (Scheme 1). Although there is a few reports on the synthesis of benzochromene based dicyanoanilines, ${ }^{13,30}$ as far as we know, this is the first general report on the synthesis of isochroman containing dicyanoanilines. The products are expected to have fluorescence properties to be explored in continuation of our investigations in the near future.

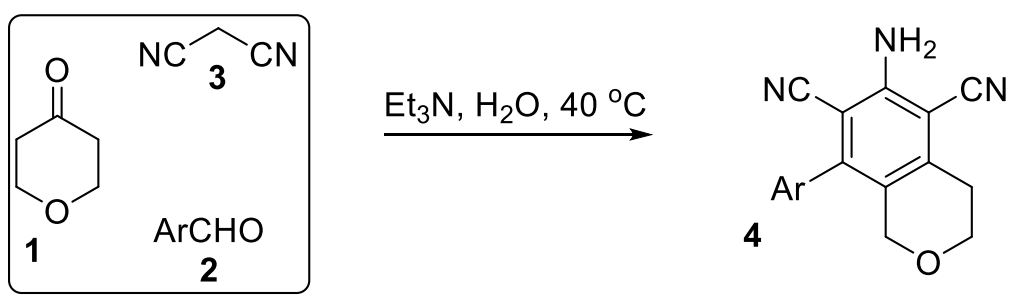

Scheme 1. One-pot synthesis of 4 .

\section{Results and Discussion}

We first optimized the process by studying the reaction of $\mathbf{1}$ with benzaldehyde $\mathbf{2} \mathbf{a}$ and $\mathbf{3}$ under various sets of conditions (Table 1). In the presence of $\mathrm{H}_{2} \mathrm{O}$ and $\mathrm{Et}_{3} \mathrm{~N}$, an equimolar mixture of $\mathbf{1}$ and the aldehyde and two folds of $\mathbf{3}$ produced $\mathbf{4 a}$ in $94 \%$ yield after $6 \mathrm{~h}$ at 40 ${ }^{\circ} \mathrm{C}$ (entry 1 ). In the absence of water (entry 2 ) or the amine (entry 3 ), either very low yield of $4 \mathbf{a}$ was obtained or the reaction was stopped completely after the same time. Alternatively, it was shown that lower temperatures gave lower quantities of $\mathbf{4 a}$ even after a prolonged time period (entry 4). Similarly, the reaction was not complete when $\mathrm{Et}_{3} \mathrm{~N}$ was used less than the optimized amounts (entries 5-7). Again, lower yields of the product was 
obtained when secondary (entries 8-9) or primary (entry 10) amines were used. The results supported that the optimum reaction is attainable when a tertiary amine is employed, as was further confimed by the use of DABCO in the reaction producing comparable amounts of $\mathbf{4 a}$ (entry 11).

Table 1. Optimization of the synthesis of $\mathbf{4 a}(\mathrm{Ar}=\mathrm{Ph})$

\begin{tabular}{|c|c|c|c|}
\hline Entry & Medium $(0.5 \mathrm{~mL})$ & Amine $(\mathrm{mol} \%)$ & Yield $(\%)^{a}$ \\
\hline 1 & $\mathrm{H}_{2} \mathrm{O}$ & $\mathrm{Et}_{3} \mathrm{~N}$ (2.0 equiv) & 94 \\
\hline 2 & - & $\mathrm{Et}_{3} \mathrm{~N}$ (2.0 equiv) & 17 \\
\hline 3 & $\mathrm{H}_{2} \mathrm{O}$ & - & 0 \\
\hline 4 & $\mathrm{H}_{2} \mathrm{O}$ & $\mathrm{Et}_{3} \mathrm{~N}(2.0 \text { equiv })^{\mathrm{b}}$ & 55 \\
\hline 5 & $\mathrm{H}_{2} \mathrm{O}$ & $\mathrm{Et}_{3} \mathrm{~N}$ (1.5 equiv) & 61 \\
\hline 6 & $\mathrm{H}_{2} \mathrm{O}$ & $\mathrm{Et}_{3} \mathrm{~N}$ (1.0 equiv) & 57 \\
\hline 7 & $\mathrm{H}_{2} \mathrm{O}$ & $\mathrm{Et}_{3} \mathrm{~N}(0.5$ equiv $)$ & 40 \\
\hline 8 & $\mathrm{H}_{2} \mathrm{O}$ & pyrrolidine ( 2.0 equiv) & 67 \\
\hline 9 & $\mathrm{H}_{2} \mathrm{O}$ & $\mathrm{Et}_{2} \mathrm{NH}$ (2.0 equiv) & 61 \\
\hline 10 & $\mathrm{H}_{2} \mathrm{O}$ & $\mathrm{BuNH}_{2}(2.0$ equiv $)$ & 55 \\
\hline 11 & $\mathrm{H}_{2} \mathrm{O}$ & BABCO (2.0 equiv) & 90 \\
\hline
\end{tabular}

${ }^{a}$ yields determined by GC. ${ }^{b}$ room temperature.

We next studied the diversity of the method by using the best conditions (Table 1, entry 1) for the reactions of $\mathbf{1}$ with $\mathbf{3}$ and different aldehydes (Table 2). Thus, not only benzaldehyde (entry 1), but also other aldehydes bearing electron donating- (entries 2-4) and electron attracting (entries 5-6) substituents combined with 1 and $\mathbf{3}$ to produce $85-95 \%$ of the respective products within 5-7 h. Similarly, furan-2-carbaldehyde (entry 7) and formaldehyde (entry 8) produced $\mathbf{4 g}$-h efficiently. In all case, products precipitated in the reaction mixtures spontaneously upon cooling. This allowed an easy separation of the products by a simple filtration.

The structure of the products was elucidated based on spectroscopic methods. In the IR spectra, two peaks appearing at about 2200 and $3300 \mathrm{~cm}^{-1}$ were in favor of the existence of the nitrile and amine groups in the final structure, respectively. In addition, in NMR spectra (both ${ }^{1} \mathrm{H}$ and ${ }^{13} \mathrm{C}$ ), three signals attributing to chemically different methylene groups confirmed the participation of the pyran ring in the structure of the target products. 
Table 2. $\mathrm{H}_{2} \mathrm{O} / \mathrm{Et}_{2} \mathrm{NH}$ catalyzed one-pot synthesis of compounds 4

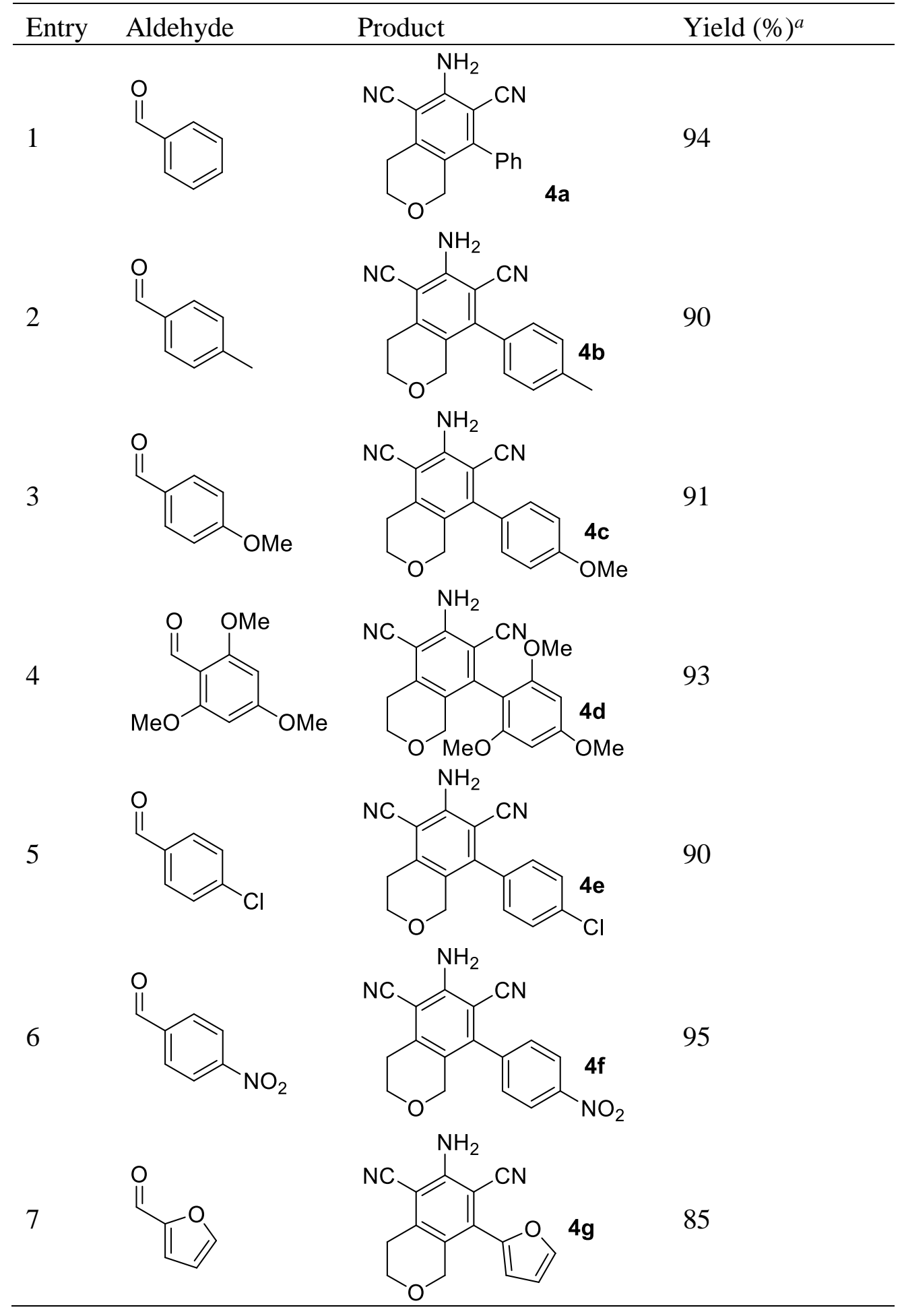


Table 2. Continued)

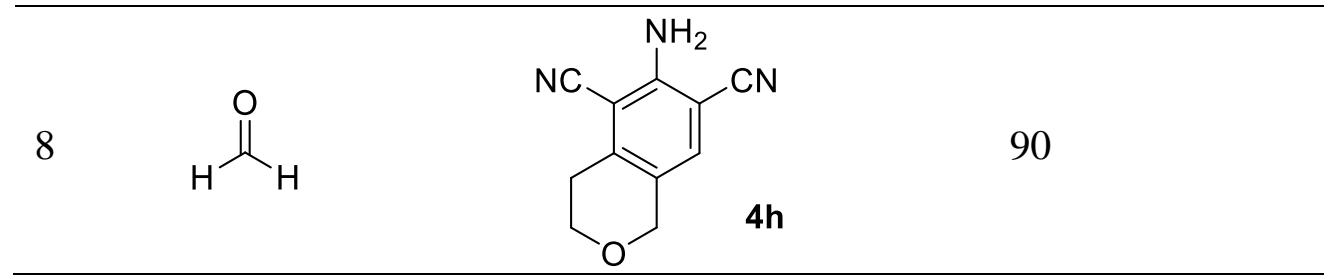

${ }^{a}$ isolated yields.

It is usual for aqueous conditioned organic reactions to be boosted by either hydrophobic ${ }^{31}$ or hydrogen bonding ${ }^{32}$ interactions. In order to see if such forces have effect on the progress of the present procedure, we conducted a group of experiments for the combination of ketone $\mathbf{1}$ with benzaldehyde and $\mathbf{3}$ (Table 3). All experiments were stopped earlier after $2 \mathrm{~h}$ so that the results can be meaningfully compared with those obtained under the optimized conditions. Entry 1 shows that when the $\mathrm{H}_{2} \mathrm{O} / \mathrm{Et}_{2} \mathrm{NH}$ conditions are used only $55 \%$ of $4 \mathbf{a}$ is obtained. Without water, the reaction rate is dramatically decreased illustrating that the aqueous medium clearly enhances the reaction (entry 2). When aqueous solutions of $\mathrm{NaCl}$ (entries 3-4) and $\mathrm{LiCl}$ (entries 5-6) were used, again a reduction in the yield of $\mathbf{4 a}$ was observed.

Table 3. Effect of different additives on the synthesis of $\mathbf{4 a}$

\begin{tabular}{cccc}
\hline Entry & Additive & Time (h) & Yield (\%) \\
\hline 1 & $\mathrm{H}_{2} \mathrm{O}$ & 2 & 55 \\
2 & - & 2 & 0 \\
3 & $\mathrm{NaCl}(1.5 \mathrm{M})$ & 2 & 20 \\
4 & $\mathrm{NaCl}(3.0 \mathrm{M})$ & 2 & 25 \\
5 & $\mathrm{LiCl}(1.5 \mathrm{M})$ & 2 & 25 \\
6 & $\mathrm{LiCl}^{(3.0 \mathrm{M})}$ & 2 & 0 \\
7 & $\mathrm{LiClO}_{4}(1.5 \mathrm{M})$ & 2 & 78 \\
8 & $\mathrm{LiClO}_{4}(3.0 \mathrm{M})$ & 2 & 81 \\
9 & $\mathrm{GnCl}_{(1.5 \mathrm{M})}$ & 2 & 63 \\
10 & $\mathrm{GnCl}_{(3.0 \mathrm{M})}$ & 2 & 72 \\
11 & $\mathrm{LiClO}_{4}(3.0 \mathrm{M})$ & 4 & 96 \\
\hline
\end{tabular}

This descending pattern which is directly proportional to the concentrations of the salts exclude the intermediacy of a "salt-out"33 effect and thus hydrophobic interactions can not have a major effect in catalyzing the process. In contrast, when $\mathrm{LiClO}_{4}$ (entries 7-8) or guanidinium chloride (entries 9-10) were used as additives, the efficiency of the reaction 
was increased and higher yields of $\mathbf{4 a}$ were obtained after the $2 \mathrm{~h}$ time period. Finally, it took only $4 \mathrm{~h}$ for the reaction to reach to $96 \%$ yield in the presence of $\mathrm{LiClO}_{4}$ solution (entry 11). This enhancement would suggest the presence of a promoting hydrogen-bonded interaction between the reactants with water to ease up the energy barrier of the process similar to what occurs in a Lewis acid mediated reaction.

Based on our study, we propose an organocatalyzed cycle, as shown in Figure 1 for the combination of $\mathbf{1}$ with benzaldehyde $\mathbf{2 a}$ and $\mathbf{3}$. In the first step, via two parallel Knoevenagel pathways, the ketone and the aldehyde undergo condensation with $\mathbf{3}$ to produce the alkene intermediates $\mathbf{1 3}$ and $\mathbf{2 3}$. Then, the amine facilitates nucleophilic combination of $\mathbf{1 3}$ and $\mathbf{2 3}$ to cause two Michael additions in a row and form intermediate $\mathbf{4 a}$ '. Tautomerization of this imine to the corresponding enamine $\mathbf{4 a "}$ and finally elimination step gives the desired product $\mathbf{4 a}$. To support the mechanism, intermediates $\mathbf{1 3}$ and 23 were prepared separately and when reacted together under the optimized conditions 4a was obtained in high yield after the expected time.

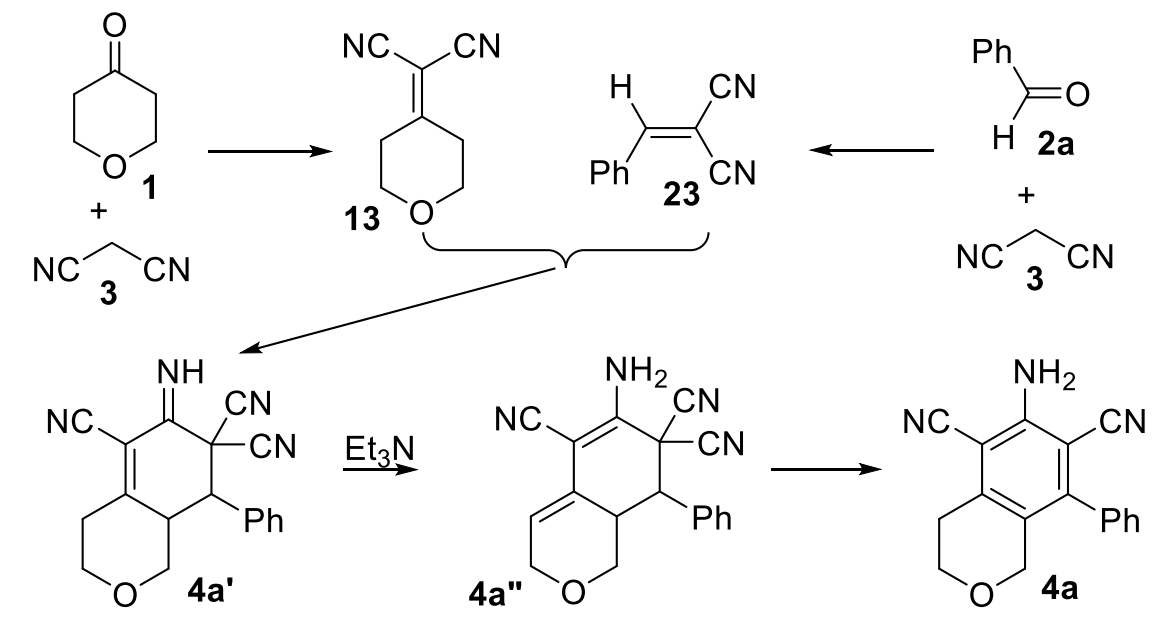

Figure 1. The proposed mechanism.

\section{Conclusions}

In summary, we succeeded in presenting an efficient one-pot procedure for the synthesis of a novel series of dicyanoanilines fused to pyran ring. Reactions took place rapidly and high yields of isochroman derivatives were obtained under very mild conditions. The extension of the method to other heterocyclic systems and the study of the optical properties of the products are underway in the near future in our laboratory. 


\section{Experimental Section}

General. Melting points are uncorrected. FT-IR spectra were recorded using $\mathrm{KBr}$ disks on a Shimadzu Prestige-21 spectrometer. NMR spectra were obtained on a FT-NMR Bruker Ultra Shield ${ }^{\mathrm{TM}}(500 \mathrm{MHz})$ as $\mathrm{CDCl}_{3}$ solutions using TMS as internal standard reference. Elemental analyses were performed using a Thermo Finnigan Flash EA 1112 instrument. MS spectra were obtained on a Fisons 8000 Trio instrument at ionization potential of 70 $\mathrm{eV}$. TLC experiments were carried out on pre-coated silica gel plates using petroleum ether/EtOAc (4:1) as the eluent. Reagents and starting materials were purchased from commercial sources. Aldehydes were redistilled or recrystallized before being used. All products are new and were identified based on their physical and spectral properties. The Knoevenagel intermediates $\mathbf{1 3}$ and $\mathbf{2 3}$ were synthesized using a known procedure. ${ }^{34}$

Typical procedure. A mixture of ketone $\mathbf{1}(200 \mathrm{mg}, 2.0 \mathrm{mmol})$, benzaldehyde $\mathbf{2 a}(203 \mu \mathrm{L}$, $2.0 \mathrm{mmol}$ ), and malononitrile $3(264 \mathrm{mg}, 4.0 \mathrm{mmol})$ in $\mathrm{H}_{2} \mathrm{O} / \mathrm{Et}_{3} \mathrm{~N}(5.0 \mathrm{~mL} / 557 \mu \mathrm{L}, 4.0$ mmol) was stirred at $40{ }^{\circ} \mathrm{C}$ for $6 \mathrm{~h}$ until TLC showed complete disappearance of the starting materials. Product 4a which precipitated spontaneously at the end of the reaction was separated by filtration and recrystallized using EtOAc/hexane mixture. The product was obtained in $94 \%$ yield $(517 \mathrm{mg})$. The product was identified based on its physical and spectral characteristics.

6-Amino-8-phenylisochroman-5,7-dicarbonitrile (4a). $\mathrm{Mp}$ 214-215 ${ }^{\circ} \mathrm{C}$; $\mathrm{IR}\left(\mathrm{KBr}, \mathrm{cm}^{-1}\right)$ 3350, 2216, 1566; ${ }^{1} \mathrm{HNMR}\left(\mathrm{CDCl}_{3}, 500 \mathrm{MHz}\right) \delta$ 7.41-7.64 (m, 3H), 7.17 (dd, J 7.8, $2.1 \mathrm{~Hz}$, $2 \mathrm{H}), 5.38(\mathrm{~s}, 2 \mathrm{H}), 4.24(\mathrm{~s}, 2 \mathrm{H}), 3.90(\mathrm{t}, J 5.8 \mathrm{~Hz}, 2 \mathrm{H}), 2.96(\mathrm{t}, J 5.8 \mathrm{~Hz}, 2 \mathrm{H}) \mathrm{ppm} ;{ }^{13} \mathrm{C} \mathrm{NMR}$ $\left(\mathrm{CDCl}_{3}, 125 \mathrm{MHz}\right) \delta 151.1,147.9,143.7,135.5,129.7,129.3,128.3,123.9,115.7,115.2$, 97.0, 96.8, 66.6, 64.3, $28.5 \mathrm{ppm}$; MS (70 ev): m/z (\%) $275\left(\mathrm{M}^{+}, 11\right), 272$ (100), 244 (53), 205 (40), 115 (86). HRMS Calcd for $\mathrm{C}_{17} \mathrm{H}_{13} \mathrm{~N}_{3} \mathrm{O}: 275.10443$, Found: 275.10721.

6-Amino-8-p-tolylisochroman-5,7-dicarbonitrile (4b). $\mathrm{Mp}$ 211-212 ${ }^{\circ} \mathrm{C}$; $\mathrm{IR}\left(\mathrm{KBr}, \mathrm{cm}^{-1}\right)$ 3350, 2216, 1571; ${ }^{1} \mathrm{HNMR}\left(\mathrm{CDCl}_{3}, 500 \mathrm{MHz}\right) \delta 7.31(\mathrm{~d}, J 8.3 \mathrm{~Hz}, 2 \mathrm{H}), 7.14(\mathrm{~d}, J 8.3 \mathrm{~Hz}$, 2H), $5.16(\mathrm{~s}, 2 \mathrm{H}), 4.35(\mathrm{~s}, 2 \mathrm{H}), 3.99(\mathrm{t}, J 5.8 \mathrm{~Hz}, 2 \mathrm{H}), 3.05(\mathrm{t}, J 5.8 \mathrm{~Hz}, 2 \mathrm{H}), 2.46(\mathrm{~s}, 3 \mathrm{H})$ ppm; ${ }^{13} \mathrm{C}$ NMR $\left(\mathrm{CDCl}_{3}, 125 \mathrm{MHz}\right) \delta 150.7,148.3,143.6,139.9,132.4,130.1,128.3,124.4$, 115.7, 115.2, 97.3, 96.8, 66.8, 64.4, 28.6, $21.8 \mathrm{ppm}$; MS (70 ev): m/z (\%) $289\left(\mathrm{M}^{+}, 18\right)$, 274 (43), 242 (21), 197 (24), 115 (10); Anal. Calcd for $\mathrm{C}_{18} \mathrm{H}_{15} \mathrm{~N}_{3} \mathrm{O}$ : C, 74.72; H, 5.23; N, 14.52. found: $\mathrm{C}, 74.84 ; \mathrm{H}, 5.27 ; \mathrm{N}, 14.68$.

6-Amino-8-(4-methoxy phenyl)isochroman-5,7-dicarbonitrile (4c). Mp 238-239 ${ }^{\circ} \mathrm{C}$; IR $\left(\mathrm{KBr}, \mathrm{cm}^{-1}\right) 3354,2214,1620 ;{ }^{1} \mathrm{HNMR}\left(\mathrm{CDCl}_{3}, 500 \mathrm{MHz}\right) \delta 7.08(\mathrm{~d}, J 8.6 \mathrm{~Hz}, 2 \mathrm{H}) 6.92(\mathrm{~d}$, $J 8.6 \mathrm{~Hz}, 2 \mathrm{H}), 5.39(\mathrm{~s}, 2 \mathrm{H}), 4.23(\mathrm{~s}, 2 \mathrm{H}), 3.87(\mathrm{t}, J 5.8 \mathrm{~Hz}, 2 \mathrm{H}), 3.79(\mathrm{~s}, 3 \mathrm{H}), 2.92(\mathrm{t}, J 5.8$ $\mathrm{Hz}, 2 \mathrm{H}) \mathrm{ppm} ;{ }^{13} \mathrm{C} \mathrm{NMR}\left(\mathrm{CDCl}_{3}, 125 \mathrm{MHz}\right) \delta 160.6,151.1,147.9,143.5,129.8,127.5$, 124.1, 115.9, 115.3, 114.7, 97.2, 96.5, 66.7, 64.4, 55.7, 28.5 ppm; Anal. Calcd for $\mathrm{C}_{18} \mathrm{H}_{15} \mathrm{~N}_{3} \mathrm{O}_{2}$ : C, 70.81; H, 4.95; N, 13.76. found: $\mathrm{C}, 70.32 ; \mathrm{H}, 4.93 ; \mathrm{N}, 13.87$.

6-Amino-8-(2,4,6-trimethoxyphenyl)isochroman-5,7-dicarbonitrile (4d). Mp 234-235 ${ }^{\circ} \mathrm{C} ; \mathrm{IR}\left(\mathrm{KBr}, \mathrm{cm}^{-1}\right)$ 3350, 2222, 1600; ${ }^{1} \mathrm{HNMR}\left(\mathrm{CDCl}_{3}, 500 \mathrm{MHz}\right) \delta 6.24$ (s, 2H), $5.04(\mathrm{~s}$, 
2H), 4.29 (s, 2H), $3.98(\mathrm{t}, J 5.7 \mathrm{~Hz}, 2 \mathrm{H}), 3.90(\mathrm{~s}, 3 \mathrm{H}), 3.78(\mathrm{~s}, 6 \mathrm{H}), 3.03(\mathrm{t}, J 5.7 \mathrm{~Hz}, 2 \mathrm{H})$ ppm; ${ }^{13} \mathrm{C} \mathrm{NMR}\left(\mathrm{CDCl}_{3}, 125 \mathrm{MHz}\right) \delta 163.1,158.2,150.5,142.8,142.0,126.5,116.0,115.6$, 105.0, 99.2, 96.7, 91.3, 66.4, 64.4, 56.2, 55.8, $28.5 \mathrm{ppm} ; \mathrm{MS}(70 \mathrm{ev}): \mathrm{m} / \mathrm{z}(\%) 365\left(\mathrm{M}^{+}\right.$, 49), 334 (61), 306 (10), 197 (17), 168 (28); Anal. Calcd for $\mathrm{C}_{20} \mathrm{H}_{19} \mathrm{~N}_{3} \mathrm{O}_{4}$ : C, 65.74; H, 5.24; N, 11.50. found: C, 66.12; H, 5.01; N, 11.22.

6-Amino-8-(4-chlorophenyl)isochroman-5,7-dicarbonitrile (4e). Mp 238-239 ${ }^{\circ} \mathrm{C}$; IR $\left(\mathrm{KBr}, \mathrm{cm}^{-1}\right) 3365,2212,1600 ;{ }^{1} \mathrm{HNMR}\left(\mathrm{CDCl}_{3}, 500 \mathrm{MHz}\right) \delta 7.35(\mathrm{~d}, J 6.5 \mathrm{~Hz}, 2 \mathrm{H}), 7.07$ $(\mathrm{d}, J 6.5 \mathrm{~Hz}, 2 \mathrm{H}), 5.53(\mathrm{~s}, 2 \mathrm{H}), 4.15(\mathrm{~s}, 2 \mathrm{H}), 3.83(\mathrm{t}, J 5.8 \mathrm{~Hz}, 2 \mathrm{H}), 2.88(\mathrm{t}, J 5.8 \mathrm{~Hz}, 2 \mathrm{H})$ ppm; ${ }^{13} \mathrm{C} \mathrm{NMR}\left(\mathrm{CDCl}_{3}, 125 \mathrm{MHz}\right) \delta 151.2,146.4,143.9,135.8,133.9,129.8,129.6,123.5$, 115.5, 115.1, 97.1, 96.7, 66.4, 64.3, 28.4 ppm; MS (70 ev): m/z (\%) $311(\mathrm{M}+2,10), 309$ $\left(\mathrm{M}^{+}, 30\right), 243$ (46), 215 (27), 151 (65), 115 (56); Anal. Calcd for $\mathrm{C}_{17} \mathrm{H}_{12} \mathrm{ClN}_{3} \mathrm{O}$ : C, 65.92; $\mathrm{H}, 3.91 ; \mathrm{N}, 13.57$. found: C, 65.68; H, 4.06; N, 13.79.

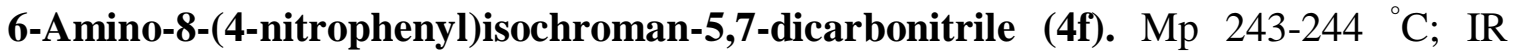
$\left(\mathrm{KBr}, \mathrm{cm}^{-1}\right) 3340,2214,1571 ;{ }^{1} \mathrm{HNMR}\left(\mathrm{CDCl}_{3}, 500 \mathrm{MHz}\right) \delta 8.31(\mathrm{~d}, J 8.5 \mathrm{~Hz}, 2 \mathrm{H}), 7.40$ (d, J $8.5 \mathrm{~Hz}, 2 \mathrm{H}), 5.61(\mathrm{~s}, 2 \mathrm{H}), 4.19(\mathrm{~s}, 2 \mathrm{H}), 3.90(\mathrm{t}, J 5.7 \mathrm{~Hz}, 2 \mathrm{H}), 2.97(\mathrm{t}, J 5.7 \mathrm{~Hz}, 2 \mathrm{H})$ ppm; ${ }^{13} \mathrm{C} \mathrm{NMR}\left(\mathrm{CDCl}_{3}, 125 \mathrm{MHz}\right) \delta 156.3,153.4,149.8,149.2,147.0,134.7,129.3,127.6$, 120.1, 119.7, 102.5, 100.9, 71.0, 69.0, $33.2 \mathrm{ppm}$; MS (70 ev): m/z (\%) $320\left(\mathrm{M}^{+}, 8\right), 244$ (11), 215 (12), 188 (15); Anal. Calcd for $\mathrm{C}_{17} \mathrm{H}_{12} \mathrm{~N}_{4} \mathrm{O}_{3}$ : C, 63.75; H, 3.78; N, 17.49. found: C, 63.64; H, 3.75; N, 16.98 .

6-Amino-8-(furan-2-yl)isochroman-5,7-dicarbonitrile (4g). Mp 210-211 ${ }^{\circ} \mathrm{C}$; IR ( $\mathrm{KBr}$, $\left.\mathrm{cm}^{-1}\right) 3348,2212,1560 ;{ }^{1} \mathrm{HNMR}\left(\mathrm{CDCl}_{3}, 500 \mathrm{MHz}\right) \delta 7.49(\mathrm{dd}, J 5.0,0.9 \mathrm{~Hz}, 1 \mathrm{H}), 7.12(\mathrm{dd}$, $J$ 5.0, 3.6 Hz, 1H), $7.05(\mathrm{dd}, J 3.5,0.9 \mathrm{~Hz}, 1 \mathrm{H}), 5.38(\mathrm{~s}, 2 \mathrm{H}), 4.41(\mathrm{~s}, 2 \mathrm{H}), 3.92(\mathrm{t}, J 5.8 \mathrm{~Hz}$, 2H), $2.97(\mathrm{t}, J 5.8 \mathrm{~Hz}, 2 \mathrm{H}) \mathrm{ppm} ;{ }^{13} \mathrm{C} \mathrm{NMR}\left(\mathrm{CDCl}_{3}, 125 \mathrm{MHz}\right) \delta 151.0,143.7,140.5,134.6$, $129.3,128.6,128.0,125.3,115.6,115.0,98.0,97.6,66.8,64.3,28.5 \mathrm{ppm} ; \mathrm{MS}(70 \mathrm{ev}): \mathrm{m} / \mathrm{z}$ (\%) $265\left(\mathrm{M}^{+}, 3\right), 248$ (100), 236 (35), 197 (64); Anal. Calcd for $\mathrm{C}_{15} \mathrm{H}_{11} \mathrm{~N}_{3} \mathrm{O}_{2}$ : C, 67.92; $\mathrm{H}$, 4.18; N, 15.84 found: C, 67.61; H, 4.52; N, 15.75 .

6-Aminoisochroman-5,7-dicarbonitrile (4h). Mp 193-194 ${ }^{\circ} \mathrm{C}$; IR (KBr, cm$\left.{ }^{-1}\right)$ 3346, 2220, 1600; ${ }^{1} \mathrm{HNMR}\left(\mathrm{CDCl}_{3}, 500 \mathrm{MHz}\right) \delta 7.30(\mathrm{~s}, 1 \mathrm{H}), 5.09(\mathrm{~s}, 2 \mathrm{H}), 4.66(\mathrm{~s}, 2 \mathrm{H}), 4.02$ (t, $J 5.2 \mathrm{~Hz}, 2 \mathrm{H}), 3.07(\mathrm{t}, J 5.2 \mathrm{~Hz}, 2 \mathrm{H}) \mathrm{ppm} ;{ }^{13} \mathrm{C} \mathrm{NMR}\left(\mathrm{CDCl}_{3}, 125 \mathrm{MHz}\right) \delta 150.9,143.9$, 133.3, 125.4, 116.3, 115.0, 98.1, 95.7, 66.9, 64.5, $28.1 \mathrm{ppm}$; MS (70 ev): m/z (\%) $199\left(\mathrm{M}^{+}\right.$, 100), 169 (87), 142 (28), 114 (33); Anal. Calcd for $\mathrm{C}_{11} \mathrm{H}_{9} \mathrm{~N}_{3} \mathrm{O}: \mathrm{C}, 67.92 ; \mathrm{H}, 4.18 ; \mathrm{N}, 15.84$ found: C, 67.84; H, 4.12; N, 15.77.

\section{Acknowledgement}

The Ministry of Science, Research, and Technology of Iran is gratefully acknowledged for partial financial support of this work. 


\section{References}

1. Hassan, S.; Müeller, T. J. J. Adv. Synth. Catal. 2015, 357, 617-666. http://dx.doi.org/10.1002/adsc.201400904

2. Khan, M. M.; Yousuf, R.; Khan, S.; Shafiullah. RSC Adv. 2015, 5, 57883-57905. http://dx.doi.org/10.1039/C5RA08059B

3. Mondal, A.; Mukhopadhyay, C. ACS Comb. Sci. 2015, 17, 404-408. http://dx.doi.org/10.1021/acscombsci.5b00038

4. Willy, B.; Müller, T. J. J. Arkivoc 2008, (i), 195-208. http://dx.doi.org/10.3998/ark.5550190.0009.107

5. Liu, C.; Zhou, L.; Jiang, D.; Gu, Y. Asian J. Org. Chem. 2016, 5, 367-372. http://dx.doi.org/10.1002/ajoc.201500497

6. Cioc, R. C.; Ruijter, E.; Orru, R. V. A. Green Chem. 2014, 16, 2958-2975. http://dx.doi.org/10.1039/C4GC00013G

7. Gu, Y. Green Chem. 2012, 14, 2091-2128. http://dx.doi.org/10.1039/C2GC35635J

8. Oshima, J.; Yoshihara, T.; Tobita, S. Chem. Phys. Lett. 2006, 423, 306-311. http://dx.doi.org/10.1016/j.cplett.2006.03.073

9. Kolek, P.; Pirowska, K.; Chacaga, L.; Najbar, J. Phys. Chem. Chem. Phys. 2003, 5, 4096-4107. http://dx.doi.org/10.1039/B305797F

10. Ramulu, B. J.; Chanda, T.; Chowdhury, S.; Nandi, G. C.; Singh, M. S. RSC Adv. 2013, 3, 5345-5349 http://dx.doi.org/10.1039/c3ra40450a

11. Yalçın, E.; Kutlu, Y. C.; Korkmaz, V.; Şahin, E.; Seferoğlu, Z. Arkivoc 2015, (v), 202-218. http://dx.doi.org/10.3998/ark.5550190.p009.102

12. Sawargave, S. P.; Kudale, A. S.; Deore, J. V.; Bhosale, D. S.; Divse, J. M.; Chavan, S. P.; Borate, H. B. Tetrahedron Lett. 2011, 52, 5491-5493. http://dx.doi.org/10.1016/j.tetlet.2011.08.064

13. Hammam, A. G.; Fahmy, A. F. M.; Amr, A. E.; Mohamed, A. M. Indian J. Chem. 2003, 42B, 1985-1993.

14. Zhang, M.-M.; Wu, J.-R.; Zhou, J.; Wang, X.-S. Synth. Commun. 2012, 42, 599-607. http://dx.doi.org/10.1080/00397911.2010.528128

15. Bhattacharjee, S.; khan, A. T. Tetrahedron Lett. 2016, 57, 2994-2997. http://dx.doi.org/10.1016/j.tetlet.2016.05.097

16. Borate, H. B.; Kudale, A. S.; Agalave, S. G. Org. Prep. Proced. Int. 2012, 44, 467521.

http://dx.doi.org/10.1080/00304948.2012.715055 
17. Chen, W.; Lu, X.-Y. Chem. Pap. 2015, 69, 1016-1020. http://dx.doi.org/10.1515/chempap-2015-0098

18. Yi, C.; Blum, C.; Liu, S.-X.; Frei, G.; Neels, A.; Renaud, P.; Leutwyler, S.; Decurtins, S. J. Org. Chem. 2008, 73, 3596-3599. http://dx.doi.org/10.1021/jo800260b

19. Xin, X.; Wang, Y.; Xu, W.; Lin, Y.; Duan, H.; Dong, D. Green Chem. 2010, 12, 893898.

http://dx.doi.org/10.1039/B921390B

20. Das, P.; Butcher, R. J.; Mukhopadhyay, C. Green Chem. 2012, 14, 1376-1387. http://dx.doi.org/10.1039/C2GC16641K

21. Yachevskii, D. S.; Chizhov, D. L.; Pashkevich, K. I.; Charushin, V. N. Arkivoc 2004, (xi), 71-76. http://dx.doi.org/10.3998/ark.5550190.0005.b10

22. Aytemir, M. D.; Çaliş, U.; Özalp, M. Arch. Pharm. Pharm. Med. Chem. 2004, 337, 281-288. http://dx.doi.org/10.1002/ardp.200200754

23. McDonald, B. R.; Scheidt, K. A. Acc. Chem. Res. 2015, 48, 1172-1183. http://dx.doi.org/10.1021/ar5004576

24. Abaee, M. S.; Akbarzadeh, E.; Shockravi, A.; Mojtahedi, M. M.; Khavasi, H. R. Can. J. Chem. 2014, 92, 659-664. http://dx.doi.org/10.1139/cjc-2013-0410

25. Mojtahedi, M. M.; Abaee, M. S.; Khakbaz, M.; Alishiri, T.; Samianifard, M.; Mesbah, A. W.; Harms, K. Synthesis 2011, 43, 3821-3826. http://dx.doi.org/10.1055/s-0031-1289571

26. Abaee, M. S.; Akbarzadeh, E.; Shockrawi, A.; Mojtahedi, M. M.; Mehraki, E.; Khavasi, H. R. Heterocycl. Commun. 2014, 20, 123-128. http://dx.doi.org/10.1515/hc-2014-0001

27. Mojtahedi, M. M.; Abaee, M. S.; Samianifard, M.; Shamloo, A. Scientia Iran 2014, $21,719-726$. http://dx.doi.org/10.1016/j.tet.2016.02.023

28. Mojtahedi, M. M.; Pourabdi, L.; Abaee, M. S.; Jami, H.; Dini, M.; Halvagar, M. R. Tetrahedron 2016, 72, 1699-1705.

29. Shestopalov, A. M.; Shestopalov, A. A.; Rodinovskaya, L. A. Synthesis 2008, 40, 125. http://dx.doi.org/10.1055/s-2007-990942

30. Sharma K. K.; David Krupadanam, G. L. Synth. Commun. 2002, 32, 1557-1562. http://dx.doi.org/10.1081/SCC-120004146

31. Gruttadauria, M.; Giacalone, F.; Marculescu, A. M.; Meo, P. L.; Riela, S.; Noto, R. Eur. J. Org. Chem. 2007, 4688-4698. http://dx.doi.org/10.1002/ejoc.200700586 
32. Blackmond, D. G.; Armstrong, A.; Coombe, V.; Wells, A. Angew. Chem., Int. Ed. 2007, 46, 3798-3800.

http://dx.doi.org/10.1002/anie.200604952

33. Zangi, R.; Hagen, M.; Berne, B. J. J. Am. Chem. Soc. 2007, 129, 4678-4686. http://dx.doi.org/10.1021/ja068305m

34. Abaee, M. S.; Mojtahedi, M. M.; Zahedi, M. M.; Khanalizadeh, G. Arkivoc 2006, (xv), 48-52.

http://dx.doi.org/10.3998/ark.5550190.0007.f06 